\title{
Focal plane complex wavefront sensing with coronagraphic phase diversity on the THD2 bench
}

\author{
Olivier Herscovici-Schiller ${ }^{\mathrm{a}}$, Laurent M. Mugnier ${ }^{\mathrm{a}}$, Pierre Baudoz ${ }^{\mathrm{b}}$, Raphaël Galicher ${ }^{\mathrm{b}}$, \\ Jean-François Sauvage ${ }^{\mathrm{a}, \mathrm{c}}$, and Baptiste Paul ${ }^{\mathrm{a},{ }^{\dagger}}$ \\ ${ }^{a}$ ONERA - The French Aerospace Lab, department of optics, F-92322 Châtillon, France \\ ${ }^{\mathrm{b}}$ LESIA, CNRS, Observatoire de Paris, Univ. Paris Diderot, UPMC, 5 place Jules Janssen, \\ F-92190 Meudon, France \\ cAix-Marseille Université, CNRS, Laboratoire d'Astrophysique de Marseille UMR 7326, \\ F-13388 Marseille, France \\ ${ }^{\dagger}$ Baptiste Paul is now with Thales Alenia Space
}

\begin{abstract}
In this article, we study the extension of COFFEE to perform an estimation of amplitude aberration from three focal plane images ; and we perform an experimental validation of pupil amplitude reconstruction with COFFEE in the very high, space-like contrast conditions of the THD2 bench of Observatoire de Paris.
\end{abstract}

Keywords: phase diversity, high contrast imaging, inverse problems, instrumentation: adaptive optics, methods: data analysis, techniques: high angular resolution, techniques: image processing.

\section{INTRODUCTION}

The final performance of current and future instruments dedicated to exoplanet detection and characterization (such as SPHERE on the VLT, GPI on Gemini North or future ground or space-based instruments) is limited by intensity residuals in the scientific image plane, which originate in uncorrected optical aberrations. The main contribution to these residuals (after correction of turbulence by Adaptive Optics for ground-based instruments) comes from the quasi-static aberrations introduced upstream of the coronagraph, which create long-lived speckles in the detector plane that can easily be mistaken for a planet. In order to reach very high contrasts such as the ones required to image Earth-like planets, these aberrations must be compensated for. We have recently developed a dedicated focal-plane wave-font sensor called COFFEE (for COronagraphic Focal-plane wave-Front Estimation for Exoplanet detection), which consists in an extension of conventional phase diversity to a coronagraphic system: aberrations both upstream and downstream of the coronagraph are estimated using two coronagraphic focal-plane images, recorded from the scientific camera itself, without any differential aberration. ${ }^{1,2}$ Such a system has been successfully validated on the SPHERE instrument, where COFFEE's estimation has been used to compensate for the phase aberration upstream of the coronagraph, leading to a contrast optimization in the whole focal plane area controlled by the AO loop. ${ }^{3}$ For planet-finder instruments that will aim at detecting and characterizing Earth-like planets from the ground or from space, several orders of magnitude in contrast must still be gained. This requires measuring and compensating not only the phase but also the amplitude aberrations (inhomogeneous intensity in the pupil, due to Fresnel propagation). In this article, we study the extension of COFFEE to perform an estimation of amplitude aberration from three focal plane images ; and we perform an experimental validation of pupil amplitude reconstruction with COFFEE in the very high, space-like contrast conditions of the THD2 bench of Observatoire de Paris.

Further author information: (Send correspondence to O.H.-S.)

O.H.-S.: E-mail: olivier.herscovici@onera.fr, Telephone: +33146734762 


\section{FORMALISM}

\subsection{Image formation model}

We consider a coronagraphic imaging system observing a point source. Let $\exp \left(\mathrm{i} \boldsymbol{\phi}_{u}+\boldsymbol{\xi}_{u}\right)$ be the complex field to be estimated in a pupil plane upstream of the coronagraph, and $\phi_{d}$ the phase aberrations in a pupil plane downstream of the coronagraph. We denote by $\boldsymbol{h}_{c}\left(\boldsymbol{\phi}_{u}, \boldsymbol{\xi}_{u}, \boldsymbol{\phi}_{d}\right)$ the model of the coronagraphic PSF as a function of $\boldsymbol{\phi}_{u}, \boldsymbol{\xi}_{u}, \boldsymbol{\phi}_{d}$. We assume that we record three coronagraphic images in the focal plane: one focused image $\boldsymbol{i}_{c}^{\text {foc }}$ and two diversity images $\phi_{\mathrm{div}_{1}}$ and $\phi_{\mathrm{div}_{2}}$, differing from the focused image by a known diversity, $\phi_{\mathrm{div}_{1}}$ and $\phi_{\mathrm{div}_{2}}$ respectively. These images can be written as:

$$
\begin{aligned}
\boldsymbol{i}_{c}^{\mathrm{foc}} & =\alpha_{\mathrm{foc}} \boldsymbol{h}_{\mathrm{det}} \star \boldsymbol{h}_{c}\left(\boldsymbol{\phi}_{u}, \boldsymbol{\xi}_{u}, \boldsymbol{\phi}_{d}\right)+n_{\mathrm{foc}}+\beta_{\mathrm{foc}} \\
\boldsymbol{i}_{c}^{\mathrm{div} 1} & =\alpha_{\mathrm{div} 1} \boldsymbol{h}_{\mathrm{det}} \star \boldsymbol{h}_{c}\left(\boldsymbol{\phi}_{u}+\boldsymbol{\phi}_{\mathrm{div} 1}, \boldsymbol{\xi}_{u}, \boldsymbol{\phi}_{d}\right)+n_{\mathrm{div} 1}+\beta_{\mathrm{div} 1} . \\
\boldsymbol{i}_{c}^{\mathrm{div} 2} & =\alpha_{\mathrm{div} 2} \boldsymbol{h}_{\mathrm{det}} \star \boldsymbol{h}_{c}\left(\boldsymbol{\phi}_{u}+\boldsymbol{\phi}_{\mathrm{div} 2}, \boldsymbol{\xi}_{u}, \boldsymbol{\phi}_{d}\right)+n_{\mathrm{div} 2}+\beta_{\mathrm{div} 2},
\end{aligned}
$$

where $\alpha_{p}$ is the flux of the star $(p \in\{$ foc, $\operatorname{div} 1, \operatorname{div} 2\}), \beta_{p}$ represents a constant residual background in image $\boldsymbol{i}_{c}^{p}$, $\boldsymbol{h}_{\mathrm{det}}$ is the detector PSF, and $\boldsymbol{n}_{p}$ denotes the measurement noise. This noise is usually a combination of photon and detector noises, which can be well approximated by a white Gaussian inhomogeneous noise for the flux levels at hand in high contrast imaging. Its variance is then the sum of the variances of the photon noise $\sigma_{\operatorname{det}_{p}}^{2}$ and of the detector noise $\boldsymbol{\sigma}_{\text {phot }_{p}}^{2}{ }^{4}$ :

$$
\boldsymbol{\sigma}_{n_{p}}^{2}[k]=\sigma_{\operatorname{det}_{p}}^{2}+\boldsymbol{\sigma}_{\text {phot }_{p}}^{2}[k]
$$

where $k$ is the current pixel index. In this equation, $\sigma_{\operatorname{det}_{p}}$ is a scalar determined by the calibration of the detector, and the variance map $\boldsymbol{\sigma}_{\text {phot }_{p}}^{2}[k]$ can be estimated simply from the image $\boldsymbol{i}_{c}^{p}$ itself $^{4}$ :

$$
\boldsymbol{\sigma}_{\text {phot }}^{2}[k]=\max \left(\boldsymbol{i}_{c}^{p}[k], 0\right)
$$

\subsection{MAP metric for phase and amplitude reconstruction}

The COFFEE method is based on the optimization of a Maximum A Posteriori (MAP) metric. It consists in searching for the upstream phase and amplitude aberrations $\hat{\boldsymbol{\phi}}_{u}$ and $\hat{\boldsymbol{\xi}}_{u}$, the downstream phase aberrations $\hat{\boldsymbol{\phi}}_{d}$, as well as the fluxes $\hat{\boldsymbol{\alpha}}=\left[\hat{\alpha}_{\text {foc }}, \hat{\alpha}_{\text {div1 }}, \hat{\alpha}_{\text {div2 }}\right]$ and the background values $\hat{\boldsymbol{\beta}}=\left[\hat{\beta}_{\text {foc }}, \hat{\beta}_{\text {div1 } 1}, \hat{\beta}_{\text {div2 } 2}\right]$ that maximize the posterior likelihood $p\left(\boldsymbol{\alpha}, \boldsymbol{\beta}, \boldsymbol{\phi}_{u}, \boldsymbol{\xi}_{u}, \boldsymbol{\phi}_{d} \mid \boldsymbol{i}_{c}^{\mathrm{foc}}, \boldsymbol{i}_{c}^{\mathrm{div} 1}, \boldsymbol{i}_{c}^{\mathrm{div} 2}\right)$. In practice, the solution is obtained by the minimization of the neg-log-likelihood metric $J$ defined as the opposite of the logarithm of this posterior likelihood, i.e.:

$$
\left(\hat{\boldsymbol{\alpha}}, \hat{\boldsymbol{\beta}}, \hat{\boldsymbol{\phi}}_{u}, \hat{\boldsymbol{\xi}}_{u}, \hat{\boldsymbol{\phi}}_{d}\right)=\underset{\boldsymbol{\alpha}, \boldsymbol{\beta}, \boldsymbol{\phi}_{u}, \boldsymbol{\xi}_{u}, \phi_{d}}{\arg \min } J\left(\boldsymbol{\alpha}, \boldsymbol{\beta}, \boldsymbol{\phi}_{u}, \boldsymbol{\xi}_{u}, \phi_{d}\right),
$$

with:

$$
\begin{aligned}
J\left(\boldsymbol{\alpha}, \boldsymbol{\beta}, \boldsymbol{\phi}_{u}, \boldsymbol{\xi}_{u}, \boldsymbol{\phi}_{d}\right)= & \frac{1}{2}\left\|\frac{\boldsymbol{i}_{c}^{\mathrm{foc}}-\left[\alpha_{\mathrm{foc}} \boldsymbol{h}_{\mathrm{det}} \star \boldsymbol{h}_{c}\left(\boldsymbol{\phi}_{u}, \boldsymbol{\xi}_{u}, \boldsymbol{\phi}_{d}\right)+\beta_{\mathrm{foc}}\right]}{\boldsymbol{\sigma}_{n_{\mathrm{foc}}}}\right\|^{2} \\
& +\frac{1}{2}\left\|\frac{\boldsymbol{i}_{c}^{\mathrm{div} 1}-\left[\alpha_{\mathrm{div} 1} \boldsymbol{h}_{\mathrm{det}} \star \boldsymbol{h}_{c}\left(\boldsymbol{\phi}_{u}+\boldsymbol{\phi}_{\mathrm{div} 1}, \boldsymbol{\xi}_{u}, \boldsymbol{\phi}_{d}\right)+\beta_{\mathrm{div} 1}\right]}{\boldsymbol{\sigma}_{n_{\mathrm{div} 1}}}\right\|^{2} \\
& +\frac{1}{2}\left\|\frac{\boldsymbol{i}_{c}^{\mathrm{div} 2}-\left[\alpha_{\mathrm{div} 2} \boldsymbol{h}_{\mathrm{det}} \star \boldsymbol{h}_{c}\left(\boldsymbol{\phi}_{u}+\boldsymbol{\phi}_{\mathrm{div} 2}, \boldsymbol{\xi}_{u}, \boldsymbol{\phi}_{d}\right)+\beta_{\mathrm{div} 2}\right]}{\boldsymbol{\sigma}_{n_{\mathrm{div} 2}}}\right\|^{2} \\
& +\mathcal{R}\left(\boldsymbol{\phi}_{u}\right)+\mathcal{R}\left(\boldsymbol{\xi}_{u}\right)+\mathcal{R}\left(\boldsymbol{\phi}_{d}\right),
\end{aligned}
$$

where $\mathcal{R}\left(\phi_{q}\right)$ represents the regularization term on the phase aberration $\phi_{q}$, with $q \in[u, d]$, and $\mathcal{R}\left(\boldsymbol{\xi}_{u}\right)$ represents the regularization term on the amplitude aberrations $\boldsymbol{\xi}_{u}$. The minimization of metric $J$ (Equation 5 ) is performed by means of the VMLM-B method, ${ }^{5}$ and relies on the analytical expressions of gradients $\partial J / \partial \phi_{u}, \partial J / \partial \boldsymbol{\xi}_{u}$ $\partial J / \partial \boldsymbol{\phi}_{d}, \partial J / \partial \boldsymbol{\alpha}$ and $\partial J / \partial \boldsymbol{\beta}$. 


\subsection{Choice of appropriate basis and regularization for the aberrations}

The use of a pixel basis for the phase reconstruction is required for COFFEE to estimate high order aberrations. However, this leads to a large number of unknowns, which in turn calls for a regularization metric in order to reduce the noise sensitivity. We chose a regularization metric that is based on the available a priori knowledge on the quasi-static aberrations. Indeed, they can be reasonably assumed to be Gaussian, homogeneous and thus endowed with a power spectral density (PSD). In this communication, we use the regularizations in $1 / f^{2}$ and $1 / f^{4}$ whose implementations are described in Ref. 2, along with a Tikhonov regularization in $1 / f^{0}$.

\section{EXPERIMENTAL TESTBED}

\subsection{The THD2 bench}

The THD2 bench is the very high contrast testbed of Observatoire de Paris. It is represented on Fig. 1.

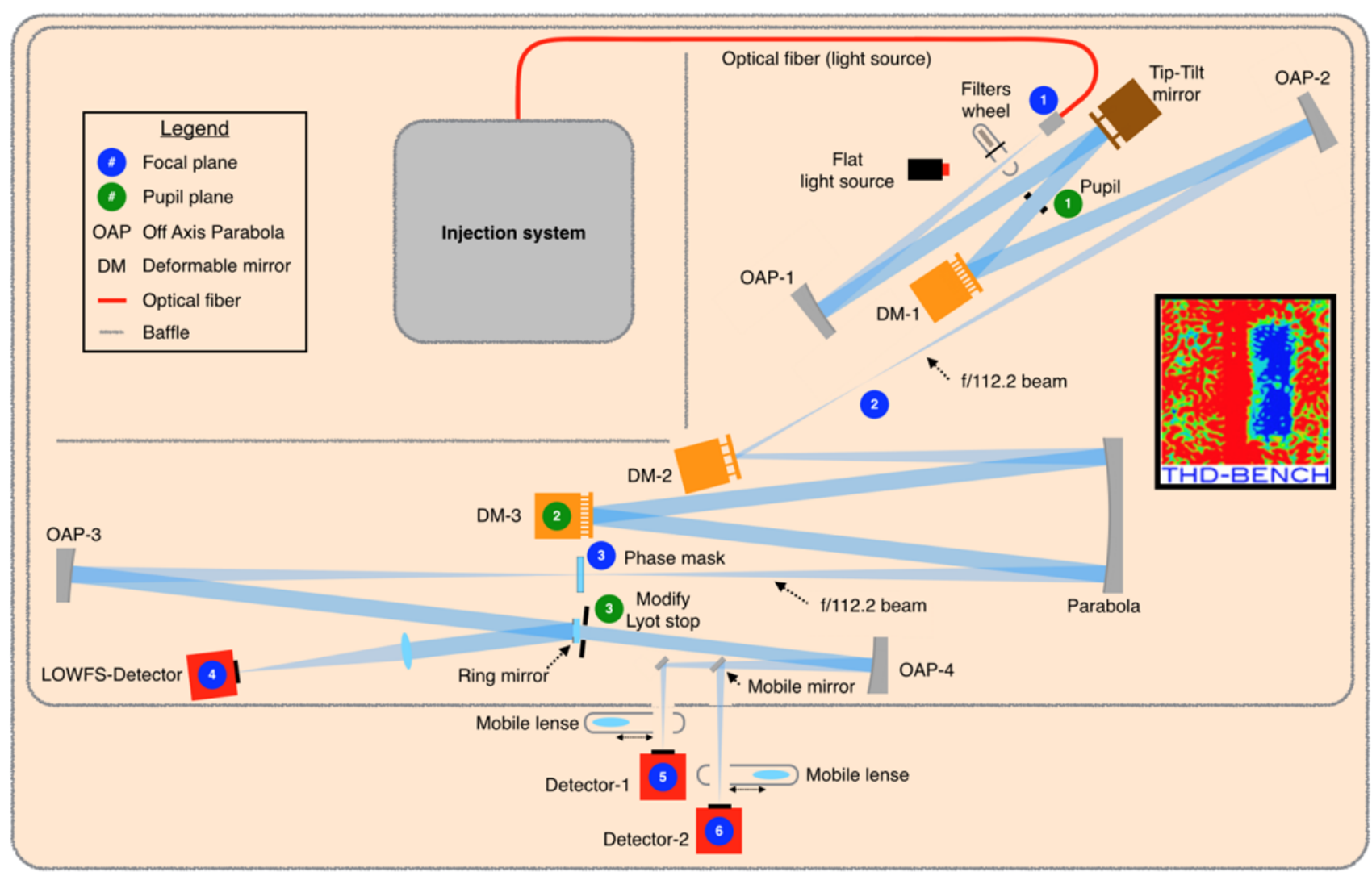

Figure 1. Optical design of the THD2 Testbed

is schematically described in Fig. 2. We will be mostly interested in the fact that it allows for the use of:

- a monochromatic light source of wavelength $783.25 \mathrm{~nm}$;

- a photometer for a precise normalization of the light flux;

- an off-pupil deformable mirror, DM1, hereafter called "amplitude mirror";

- a pupil-plane deformable mirror, DM3, hereafter called "phase mirror";

- a four-quadrant phase mask coronagraph;

- a camera. 


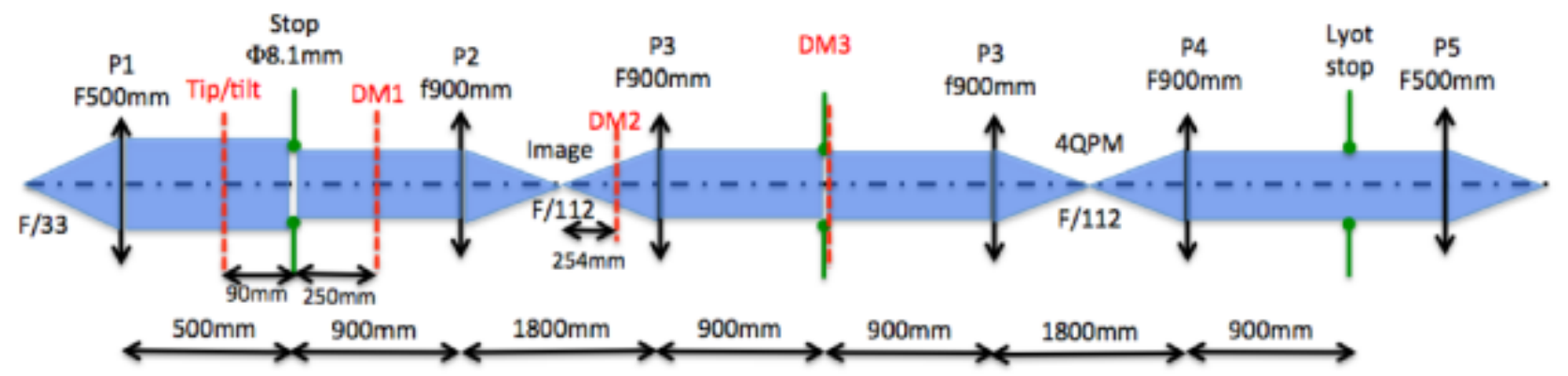

Figure 2. Schematic design of the THD2 Testbed

\section{AMPLITUDE RETRIEVAL USING COFFEE}

The goal of this paper is to demonstrate the ability of COFFEE to estimate correctly amplitude defects, in a very high contrast set-up. We do this in three steps.

The first step is the estimation of a reference wave-front, to calibrate the static aberration of the bench. The second step is to introduce a known amplitude aberration, then estimate this new wavefront. The third step is to make the difference and compare with the command on the mirror.

\subsection{Reference wavefront}

The phase and amplitude mirror are controlled using an SCC closed-loop in order to obtain a contrast of $2 \times 10^{-8}$ in the focal plane. The corresponding wavefront upstream of the coronagraph is $\left(\phi_{u}^{0}, \boldsymbol{\xi}_{u}^{0}\right)$. We then acquire the focal image $\boldsymbol{i}\left(\phi_{u}^{0}, \boldsymbol{\xi}_{u}^{0}\right)$, and the two diversity images $\boldsymbol{i}\left(\phi_{u}^{0}+\phi_{\operatorname{div} 1}, \boldsymbol{\xi}_{u}^{0}\right)$ and $\boldsymbol{i}\left(\phi_{u}^{0}+\phi_{\operatorname{div} 2}, \boldsymbol{\xi}_{u}^{0}\right)$, all shown on Fig. 3 .

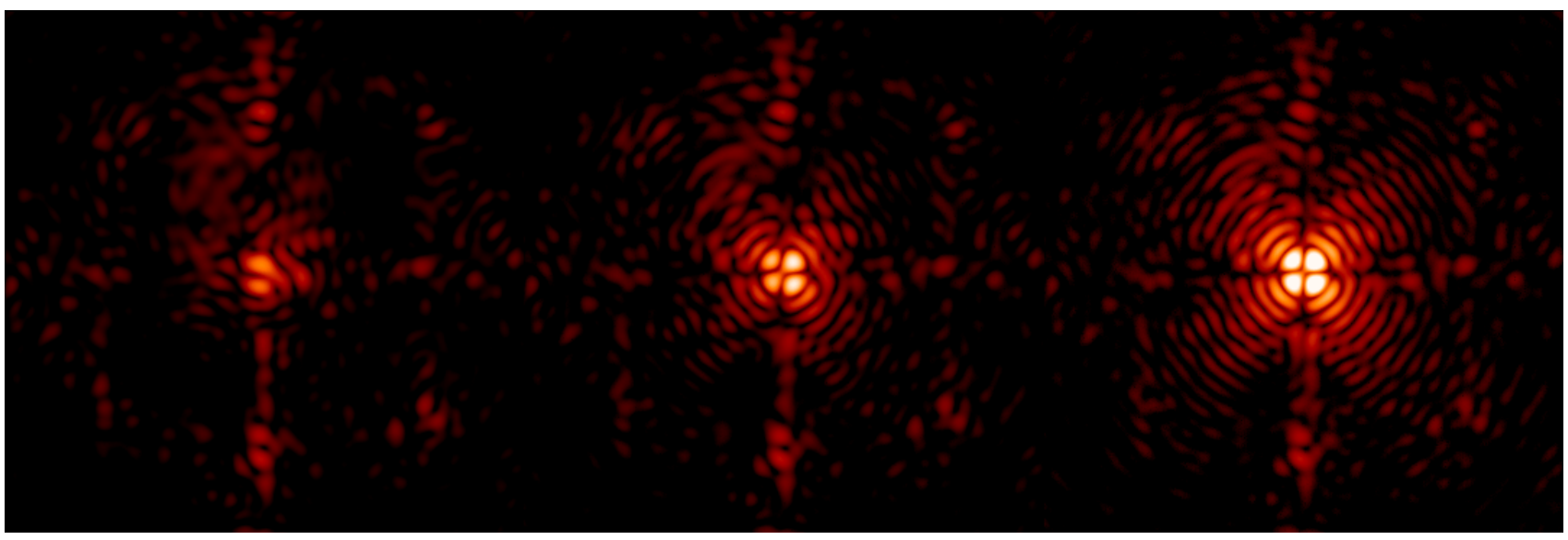

Figure 3. Focal-plane coronagraphic images with the reference wave-front. From left to right: $\boldsymbol{i}\left(\boldsymbol{\phi}_{u}^{0}, \boldsymbol{\xi}_{u}^{0}\right), \boldsymbol{i}\left(\boldsymbol{\phi}_{u}^{0}+\boldsymbol{\phi}_{\mathrm{div} 1}, \boldsymbol{\xi}_{u}^{0}\right)$, and $\boldsymbol{i}\left(\phi_{u}^{0}+\phi_{\mathrm{div} 2}, \boldsymbol{\xi}_{u}^{0}\right)$.

\subsection{Wave-front with an amplitude aberration}

The second step is to act on the phase mirror and on the amplitude mirror in order to add a known aberration to the reference wave-front.

On the amplitude mirror, we add a near-horizontal sinusoid. The frequency of this sinusoid is carefully calibrated with respect to the position of the mirror so that, thanks to Talbot propagation, this off-pupil plane sinusoid is equivalent to an amplitude defect $\boldsymbol{\xi}_{u}^{a}$ with a variance of $2.0 \mathrm{~nm}$, which is accountable for the spots in the rightmost image of Fig. 4. 
The corresponding wavefront upstream of the coronagraph is $\left(\phi_{u}^{0}, \boldsymbol{\xi}_{u}^{0}+\boldsymbol{\xi}_{u}^{a}\right)$. We then acquire images $\boldsymbol{i}\left(\boldsymbol{\phi}_{u}^{0}, \boldsymbol{\xi}_{u}^{0}+\right.$ $\left.\boldsymbol{\xi}_{u}^{a}\right), \boldsymbol{i}\left(\phi_{u}^{0}+\phi_{\mathrm{div} 1}, \boldsymbol{\xi}_{u}^{0}+\boldsymbol{\xi}_{u}^{a}\right)$, and $\boldsymbol{i}\left(\phi_{u}^{0}+\phi_{\mathrm{div} 2}, \boldsymbol{\xi}_{u}^{0}+\boldsymbol{\xi}_{u}^{a}\right)$ shown on Fig. 4 .

Our objective will be to reconstruct $\phi_{u}^{0}$ and $\boldsymbol{\xi}_{u}^{0}$ using only the focal-plane images $\boldsymbol{i}\left(\phi_{u}^{0}, \boldsymbol{\xi}_{u}^{0}\right), \boldsymbol{i}\left(\phi_{u}^{0}+\phi_{\mathrm{div} 1}, \boldsymbol{\xi}_{u}^{0}\right)$, $\boldsymbol{i}\left(\phi_{u}^{0}+\phi_{\mathrm{div} 2}, \boldsymbol{\xi}_{u}^{0}\right), \boldsymbol{i}\left(\phi_{u}^{0}, \boldsymbol{\xi}_{u}^{0}+\boldsymbol{\xi}_{u}^{a}\right), \boldsymbol{i}\left(\phi_{u}^{0}+\phi_{\mathrm{div} 1}, \boldsymbol{\xi}_{u}^{0}+\boldsymbol{\xi}_{u}^{a}\right)$, and $\boldsymbol{i}\left(\phi_{u}^{0}+\phi_{\mathrm{div} 2}, \boldsymbol{\xi}_{u}^{0}+\boldsymbol{\xi}_{u}^{a}\right)$.

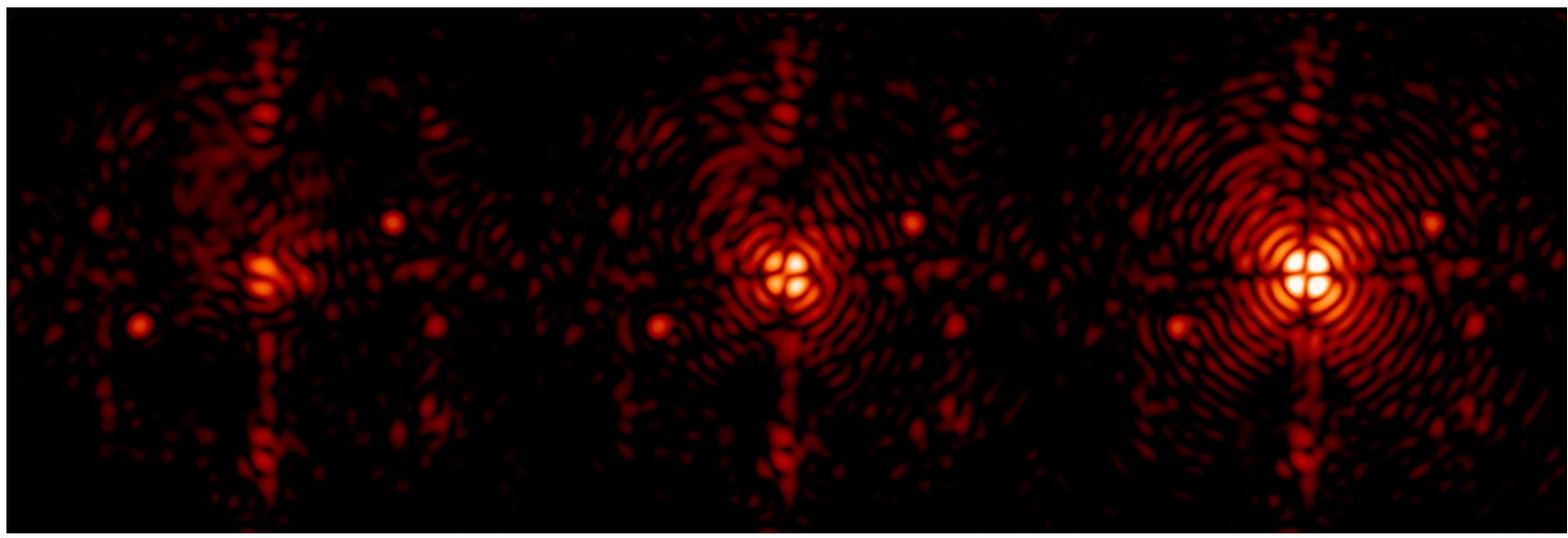

Figure 4. Focal-plane coronagraphic images with the aberrant wave-front. From left to right: $\boldsymbol{i}\left(\phi_{u}^{0}, \boldsymbol{\xi}_{u}^{0}+\boldsymbol{\xi}_{u}^{a}\right), \boldsymbol{i}\left(\phi_{u}^{0}+\right.$ $\left.\phi_{\mathrm{div} 1}, \boldsymbol{\xi}_{u}^{0}+\boldsymbol{\xi}_{u}^{a}\right)$, and $\boldsymbol{i}\left(\phi_{u}^{0}+\phi_{\mathrm{div} 2}, \boldsymbol{\xi}_{u}^{0}+\boldsymbol{\xi}_{u}^{a}\right)$.

\subsection{Amplitude retrieval with COFFEE}

We use the reference images displayed on Fig. $3 \boldsymbol{i}\left(\phi_{u}^{0}, \boldsymbol{\xi}_{u}^{0}\right), \boldsymbol{i}\left(\phi_{u}^{0}+\phi_{\mathrm{div} 1}, \boldsymbol{\xi}_{u}^{0}\right)$ and $\boldsymbol{i}\left(\phi_{u}^{0}+\phi_{\mathrm{div} 2}, \boldsymbol{\xi}_{u}^{0}\right)$ as input of COFFEE. In a few minutes*, we obtain an estimate $\left(\hat{\boldsymbol{\phi}}_{u}^{0}, \hat{\boldsymbol{\xi}}_{u}^{0}\right)$ of $\left(\boldsymbol{\phi}_{u}^{0}, \boldsymbol{\xi}_{u}^{0}\right)$.

In a similar fashion, we use the reference images displayed on Fig. $4 \boldsymbol{i}\left(\phi_{u}^{0}+, \boldsymbol{\xi}_{u}^{0}+\boldsymbol{\xi}_{u}^{a}\right), \boldsymbol{i}\left(\phi_{u}^{0}+\boldsymbol{\phi}_{\mathrm{div} 1}, \boldsymbol{\xi}_{u}^{0}+\boldsymbol{\xi}_{u}^{a}\right)$, and $\boldsymbol{i}\left(\boldsymbol{\phi}_{u}^{0}+\boldsymbol{\phi}_{\mathrm{div} 2}, \boldsymbol{\xi}_{u}^{0}+\boldsymbol{\xi}_{u}^{a}\right)$ as input of COFFEE. In a few minutes, we obtain an estimate $\left(\hat{\boldsymbol{\phi}}_{u}^{1}, \hat{\boldsymbol{\xi}}_{u}^{1}\right)$ of $\left(\boldsymbol{\phi}_{u}^{0}, \boldsymbol{\xi}_{u}^{0}+\boldsymbol{\xi}_{u}^{a}\right)$.

We then calculate the difference $\left(\hat{\boldsymbol{\xi}_{u}^{a}}\right)=\left(\hat{\phi}_{u}^{1}, \hat{\boldsymbol{\xi}}_{u}^{1}\right)-\left(\hat{\boldsymbol{\phi}_{u}^{0}}, \hat{\boldsymbol{\xi}_{u}^{0}}\right)$, which is our estimate of the aberrations that we introduced by acting on the phase mirror and the amplitude mirror. Figure 5 displays this estimate, which shows the expected sinusoidal shapes. A much more detailed analysis is to be performed in 6 .

\section{REFERENCES}

[1] Sauvage, J.-F., Mugnier, L., Paul, B., and Villecroze, R., "Coronagraphic phase diversity: a simple focal plane sensor for high-contrast imaging," Opt. Lett. 37, 4808-4810 (Dec. 2012).

[2] Paul, B., Mugnier, L. M., Sauvage, J.-F., Dohlen, K., and Ferrari, M., "High-order myopic coronagraphic phase diversity (COFFEE) for wave-front control in high-contrast imaging systems," Opt. Express 21(26), 31751-31768 (2013).

[3] Paul, B., Sauvage, J.-F., Mugnier, L. M., Dohlen, K., Petit, C., Fusco, T., Mouillet, D., Beuzit, J.-L., and Ferrari, M., "Compensation of high-order quasi-static aberrations on SPHERE with the coronagraphic phase diversity COFFEE," Astron. Astrophys. 572, A32 (Nov. 2014).

[4] Mugnier, L. M., Fusco, T., and Conan, J.-M., "MISTRAL: a myopic edge-preserving image restoration method, with application to astronomical adaptive-optics-corrected long-exposure images.," J. Opt. Soc. Am. A 21, 1841-1854 (Oct. 2004).

[5] Thiébaut, E., "Optimization issues in blind deconvolution algorithms," in [Astronomical Data Analysis II], Starck, J.-L. and Murtagh, F. D., eds., 4847, 174-183, Proc. Soc. Photo-Opt. Instrum. Eng. (Dec. 2002).

[6] Herscovici-Schille, O. et al., "Experimental validation of simultaneous phase and amplitude wave-front sensing with coronagraphic phase diversity for high-contrast imaging.," Submitted .

${ }^{*}$ Calculations were made on IDL running on a single core of an Intel Xeon CPU at $2.80 \mathrm{GHz}$. 


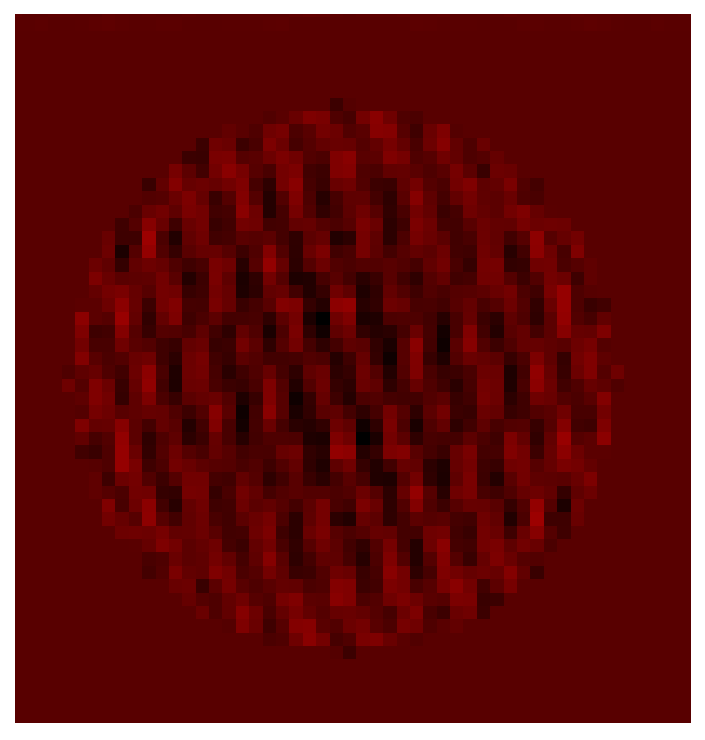

Figure 5. $\left(\hat{\boldsymbol{\xi}_{u}^{a}}\right)$ 